\title{
Aportes al objeto de conocimiento en la Especialización en Educación y Orientación Familiar de la Fundación Universitaria Monserrate ${ }^{1}$
}

\author{
Contributions to the object of knowledge in the Postgra- \\ duate Program in Education and Family Orientation of the \\ Fundación Universitaria Monserrate (Bogotá, Colombia)
}

Luz Sney Cardozo Espitia ${ }^{2}$

\section{Resumen}

El presente artículo da cuenta de los resultados de la investigación denominada Aportes que hacen los estudios de investigación en el periodo (2010-2015) a las Unidades de Significación del objeto de conocimiento de la Especialización en Educación y Orientación Familiar en el marco de la Educación Superior de la Fundación Universitaria Monserrate - Unimonserrate. La investigación se abordó desde el enfoque cualitativo con un paradigma hermenéutico que sitúo la interpretación como vínculo para la comprensión de los lenguajes y los discursos encontrados en los proyectos de investigación que realizaron los estudiantes en formación y tuvieron como objeto de estudio la familia.

Palabras clave: Familia; Comunicación; Socio afectividad; Organización.
This paper reports the results of the research work called Contributions arising from research studies in the period (2010-2015) to the Meaning Units of the knowl-edge objects of the Postgraduate program in Education and Family Orientation in the framework of Higher Education at Fundacion Universitaria Monserrate (Bo-gotá, Colombia).

The research was developed from the qualitative ap-proach with a hermeneutic paradigm that places in-terpretation as a link for the understanding of the lan-guages and discourses found in the research projects carried out by the students in training and which ob-ject of study was family

Keywords: Family; Communication; Affective partner; Organization.

${ }^{1}$ Artículo de investigación resultado de la tesis de grado Aportes que hacen los estudios de investigación en el periodo (2010-2015) a las Unidades de Significación del Objeto de Conocimiento

2 Trabajadora Social. Magister en Desarrollo Educativo y Social. Docente investigadora -Especialización en Educación y Orientación Familiar de la Fundación Universitaria Monserrate - Unimonserrate. E-mail: luzsney@gmail.com

Cómo citar este artículo: Cardozo, E. L. (2017). Aportes al objeto de conocimiento en la Especialización en Educación y Orientación Familiar de la Fundación Universitaria Mon-serrate. Revista Hojas y Hablas, (14), 154-166. doi: 10.29151/hojasyhablas.n14a12 


\section{Introducción}

Esta investigación surge en el marco de la Especialización de Educación y Orientación Familiar de la Fundación Universitaria Monserrate, la cual tiene como misión la comprensión de la función socializadora de las familias, desde un sentido humanista y una mirada sistémica para su acompañamiento, promoción y mejoramiento de la calidad de vida (Fundación Universitaria Monserrate, 2009, p. 13). Este escenario de formación pos-gradual se configura desde el campo de la Educación, con un eje fundante pedagógico denominado Orientación (Fundación Universitaria Monserrate, 2009, p. 4).

El estudio se desarrolló entre el II semestre de 2015 y I semestre de 2016 con la dinamizadoras (Asencio \& Cardozo) y los estudiantes ( Pardo, Bautista, Bayona, Medina, Bejarano, Rodríguez, Benito, Murcia, Cardona, Alarcón, Collazos, Mancilla, Cubillos, Peña, González, Medina, Jiménez, Torres, Gómez, Mantilla, Melo, Ramos, Méndez, Miranda, Gutiérrez, López, Ortiz, Gómez Ramírez, Arizmendi, Santa María, Castro, Tunjano, Valderrama , Rueda \& Virguez) de la cohorte 8 de esta especialización.

De acuerdo a lo anterior la formación en la Especialización está asociada a "la significación holística de los cualificadores (ejes trasversales en el plan de estudios) que producen un impacto cualitativo en la cultura, tanto en el objeto de estudio (familias) como en el sujeto (estudiante) y en las relaciones sujetoobjeto-contexto" (Fundación Universitaria Monserrate, 2009, p. 42). En este sentido, a través del currículo se desarrollan hipótesis que configuran y sitúan unidades de significación (Organización, Socio afectividad y Comunicación familiar) que potencian cada uno de los ejes transversales, los cuales se asumen desde la pluralidad de miradas; en este punto la comprensión y la contextualización en torno a la familia en el campo de la orientación y la educación son elementos claves para lograrlo. (Fundación Universitaria Monserrate, 2009, pp. 42-51).

En esta línea de argumentación la formación y la investigación en la Universidad son comprendidas como un proceso constitutivo fundamental. Al respecto argumenta el Documento de Renovación de Registro Calificado (Fundación Universitaria Monserrate, 2009, p. 96):

La investigación ha sido concebida en el Programa como un espacio formativo que busca hacer de ella el proceso y el estilo cognoscitivo por excelencia. El estudiante que ingresa a la Especialización, a partir de procesos teórico - prácticos, va adquiriendo las competencias para abordar, desde una perspectiva educativa, y de manera sistemática y organizada, la realidad familiar, su dinámica, relaciones, procesos, dentro del ámbito pluricultural de la sociedad actual.

En este contexto, los estudiantes en su proceso de formación profesional a partir de los saberes, las reflexiones y las problematizaciones que configuran cada uno de los cualificadores, se realiza un proyecto de investigación como opción de grado de los estudiantes de la Especialización.

Los trabajos de grado realizados (unidades de análisis de esta investigación) se articulan con la línea del programa función socializadora de la familia, y se fundamentan desde marcos epistemológicos como el enfoque sistémico y la complejidad, la cual a su vez tiene tres sublíneas: a) diversidad familiar: esta sublínea estudia la familia como instancia social, reconociendo la comprensión de 
dinámicas a través de lo organizativo en un marco estructural, lo funcional desde una perspectiva relacional y lo evolutivo desde la satisfacción de necesidades y expectativas de crecimiento en cada uno de los miembros; b) modo de atención a las familias: estudia las diferentes formas de acompañamiento a la familia, especialmente desde lo sociocultural, allí se analiza la convivencia familiar tales como el desplazamiento forzado, las migraciones, el abuso sexual entre otras; por último, la sublínea de imaginarios sobre la familia la cual sitúa la comprensión de sí misma, a la vez explica cómo actúa en el contexto que la rodea (Fundación Universitaria Monserrate, 2009, pp. 150-151).

En el marco de lo anterior, surge la investigación Aportes que hacen los estudios de investigación en el periodo (2010-2015) a las Unidades de Significación del Objeto de Conocimiento de la Especialización en Educación y Orientación $\mathrm{Fa}$ miliar, que permite comprender desde los hallazgos encontrados en cada uno de los estudios, la comprensión, la reflexión, la problematización, de las familias y sus necesidades de orientación en un contexto contemporáneo.

\section{Metodología}

Esta investigación asumió el paradigma Interpretativo entendido como afirma Khun (1986, p. 271, citado en González, 2003, p. 125): “[...] un paradigma significa una cosmovisión del mundo compartida por una comunidad científica; un modelo para situarse ante la realidad, interpretarla y dar solución a los problemas que en ella se presentan".

En este contexto, la investigación se planteó escuela hermenéutica, enfoque cualitativo, el cual aborda fenómenos a profundidad y se conduce básicamente en ambientes naturales, en los cuales los significados se extraen de los datos (Sampieri, 2010, pp. 12-14). El proceso se llevó a cabo de manera inductiva, recurrente, con análisis de múltiples realidades subjetivas y sin secuencia lineal. Según Sampieri (2010), el diseño de este tipo de investigación es abierto, flexible y no pretende generalidades, lo que facilitó el uso de datos profundos, enriquecedores y la comprensión de las personas y sus contextos, referidos en las tesis de investigación estudiadas.

Teniendo en cuenta lo anterior y conforme a los objetivos planteados, se determinó trabajar el tipo de investigación documental, considerada como no solo una técnica de recolección y validación de información, sino una estrategia cualitiativa que analiza e interpreta fuentes primarias y secundarias. (Galeano, 2004, p. 114).

Para llevarla a cabo, se tuvieron en cuenta los planteamientos de Galeano (2004) cuando refiere que "la investigación documental no requiere que el investigador participe del mundo que estudia. Por el contrario, su trabajo lo realiza desde fuera”. La estrategia de investigación, permitió la ejecución del estudio, recolectar la información, procesarla, codificarla, generar inferencias a la luz de los objetivos propuestos y realizar un profundo análisis del discurso a partir de los siguientes pasos:

a). Identificación de las fuentes de información, consultando los trabajos de investigación de la especialización en Educación y orientación familiar de la Universidad Monserrate, durante los años 2010 a 2015. En este sentido se consultaron en total 48 proyectos de investigación elaborados por los estudiantes ya egresados de la especialización entre el periodo 2010-2015. En el balance de los proyectos se encuentran los siguientes datos:

\section{Periodos}

Periodo 2006-2010: 9 Investigaciones.

Periodo 2011-2015: 39 Investigaciones.

Unidades de significación abordadas por las investigaciones

Comunicación: 12 Investigaciones. 
Socio afectividad: 7 investigaciones.

Organización Familiar: 19 investigaciones.

Comunicación y Socio afectividad: 10 investigaciones.

b) Selección de contenidos discursivos a través de la lectura detallada de cada una de las fuentes de investigación y la ubicación de citas claves para dicho análisis. Se elaboró la matriz de organización de la información (nominación del tema, problemática, población, categoría, subcategoría, dato contextual y código).

c) Segmentación del discurso por medio de la separación de las categorías encontradas, la identificación de sistemas teóricos generales y sustantivos para apoyar los procesos de significación inicial de los temas e interpretación de los datos, de la misma forma se elaboró la matriz del análisis de la información.

d) Normalización del discurso, buscando analizar la información según la organización categórica establecida, apoyándonos en la codificación axial entendida por Sampieri (2010) como la parte del análisis donde el investigador agrupa las piezas de los datos identificados y separados por el investigador en la codificación abierta, para crear conexiones entre categorías y temas. Todo esto relacionando con el fenómeno, las condiciones contextuales, causales e intervinientes, las acciones e interacciones, los resultados y las conclusiones.

e) Definiciones contextuales, abordando marcos teóricos existentes y relacionándolos con los hallazgos de la investigación a la luz de los cualificadores de formación, dando como resultado núcleos de conocimiento o tendencias de saber.

De acuerdo a todo lo anterior y por las características del estudio, las siguientes categorías de análisis, preguntas y objetivos de investigación.
Tabla 1. Categorías de análisis:

\begin{tabular}{|c|c|}
\hline Categoría "Cualificadores" & Subcategorías \\
\hline Comunicación & $\begin{array}{l}\text { - Comunicación y } \\
\text { - Colaciones humanas. } \\
\text { - Comunicación en } \\
\text { - Coreja. } \\
\text { - Comunicación } \\
\text { - Comunicación y } \\
\text { - Comientación. } \\
\text { - } \\
\text { medios masivos. }\end{array}$ \\
\hline Socio afectividad & $\begin{array}{l}\text { - Construcción de } \\
\text { vínculos. } \\
\text { - Socio afectividad } \\
\text { en las relaciones de } \\
\text { padres e hijos. } \\
\text { - Relaciones afectivas } \\
\text { en los adultos. }\end{array}$ \\
\hline Organización Familiar & $\begin{array}{l}\text { - Modos de } \\
\text { Organización } \\
\text { familiar. } \\
\text { - Estilos educativos de } \\
\text { la familia. }\end{array}$ \\
\hline
\end{tabular}

Fuente: Cardozo (2016)

\section{Pregunta de investigación}

¿Cuáles son los aportes que hacen los estudios de investigación periodo (2010-2015) a las Unidades de Significación del Objeto de Conocimiento de la Especialización de Educación y Orientación Familiar Unimonserrate?

\section{Objetivos de la Investigación}

1. Comprender los aportes de los estudios de investigación a las unidades de significación del objeto de conocimiento de EEOF.

2. Identificar los aportes - teóricos de las unidades de 
significación a los trabajos de grado de investigación Discusión y Resultados

Los resultados encontrados se presentarán dando cumplimiento a los objetivos específicos planteados por la investigación y por ende a la pregunta de indagación.

Objetivo Especifico No 1: Aportes de los estudios de investigación a las unidades de significación del objeto de conocimiento de EEOF.

Los datos se describen desde la nominación de los núcleos de conocimiento, comprendidos como tendencias de saber a partir del proceso metodológico implementado para el desarrollo del objeto de la Especialización, los cuales configuran reflexiones, aportes y problematizaciones a partir de los trabajos de grado a las Unidades de Significación.

Los núcleos encontrados y nominados fueron:

1. Imaginarios de Familia "territorios" para la comprensión de necesidades contemporáneas de formación e intervención.

2. El afecto en la relación de padres-hijos en el "Estado de emergencia".

3. La adolescencia un "territorio lleno de incertidumbre"

4. A continuación, se describen los resultados por cada uno de los núcleos de conocimiento.

\section{Núcleo de Conocimiento: Imaginarios de} Familia "territorios" para la comprensión de necesidades contemporáneas de formación e intervención.

Este núcleo de conocimiento, aporta específicamente a la sublínea de investigación de Imaginarios de Familia, que busca como su nombre lo indica conocer los imaginarios del objeto de Especialización y su función socializadora. En razón que a las tres últimas décadas se han producido cambios de tipo social, económico, cultural que tienen repercusiones en la configuración de las familias (Fundación Universitaria Monserrate,
2009, p. 151). Los imaginarios sociales, según lo argumenta Castoriadis (1989, p. 8), son:

(...) una categoría clave en la interpretación como producción de creencias e imágenes colectivas. Lo deseable, lo imaginable y lo pensable de la sociedad actual. Por lo cual, ésta se convierte en el espacio de construcción de identidades colectivas a la manera de "verse, imaginarse y pensarse como"

El término de "territorios", concepto de carácter metafórico utilizado para la nominación de este núcleo de conocimiento, según Lozano (2015) consiste en "la relación entre el sujeto y el espacio, sino fundamentalmente a las relaciones intersubjetivas ubicadas espacio-temporalmente, históricamente, cultural y socialmente" (a lo anterior se suma el concepto de terruño como escenario entrañable y vinculante que configura la pertenencia con el territorio) es decir, en el contexto de imaginarios los “territorios" hacen referencia a las realidades y representaciones de las familias, fundamentadas en sus los lenguajes y pensamientos.

De acuerdo al análisis del discurso en la investigación se encontraron los siguientes imaginarios:

\section{Relación Familia Escuela}

En esta relación se encuentran varios imaginarios que se configuran desde los roles de los protagonistas que matizan esta interacción. Según las investigaciones analizadas, "los maestros, simplifican el trabajo en lo académico, en el conocimiento, reconocen las problemáticas que existen en las familias y su afectación en la relación pedagógica, pero creen que no existen propuestas para solucionarla" (Moreno, Ospina, Velandia \& Velásquez, 2012, p. 38). En este contexto los docentes ven la función educativa relacionada directamente con el proceso de enseñanza- aprendizaje y no con la orientación 
de situaciones familiares, consideran que son competencia a otras áreas tales como Trabajo Social y Psicología.

Otro de sus imaginarios que coincide en la mayoría de las investigaciones que se estudiaron esta categoría, es que consideran que la función socializadora de la familia, es delegada a la escuela en su totalidad, esto se evidencia en la falta de conocimiento y de responsabilidad por parte de los padres al proceso educativo de los hijos, lo cual se concreta en los espacios cotidianos escolares con la ausencia de interés por parte de la familia en buscar orientación, acompañamiento, atención y solución a la problemática de abuso sexual, maltrato físico y emocional hacia sus hijos.

De acuerdo a lo anterior, se puede afirmar que la relación de Familia- Escuela, tiene repercusiones en lo afectivo, lo académico en los niños y niñas, en las relaciones de padres e hijos, relación docentes- estudiantes, docentes padres. Al respecto argumenta Bolívar, A. (2006) que las escuelas que proponen se centran en este tipo de relación tienen mayor resultado en el aprendizaje de los estudiantes, aumentanto la motivación de los profesores y mayor impacto de la escuela en la comunidad.

\section{Familia}

Los imaginarios de familia, encontrados en las investigaciones tal como lo argumentan Infante y Alayón (2013, p. 24) se ancla y prevalecen en este contexto contemporáneo a una filiación con lo se ha configurado históricamente la institucionalidad, en relación con la unión de pareja, reproducción de hijos, adquisición de bienes y la representación de juntos "para siempre".

Esto evidencia como el imaginario tal como lo expresa Castoriadis (1989), prevalece un pensamiento histórico y culturalmente construido y aún en los algunos contextos y casos es difícil de desconfigurar, resignificar o desconstruir, esto posibilita la naturalización de círculos de violencia para mantener este imaginario. Se observa entonces cómo el imaginario instituido de generación en generación en este territorio preconfigurado, se vuelve vital en los procesos de estructura y organización familiar.

En este contexto también se encuentra, una relación directa entre lo afectivo y lo económico (Infante y Alayón, 2013, p. 24), lo cual como lo argumentan estos autores se identifica en progreso en bienes materiales y la necesidad de promover su desarrollo humano a través de conseguir una vida económica que sus generaciones anteriores no tuvieron. En este sentido, es importante recalcar que la dimensión económica es un objetivo que prevalece en las familias por encima de lo afectivo, es decir, los padres luchan por un proyecto de vida económico para sus hijos relegando el área emocional y si atribuyéndoles por la ausencia en el hogar, funciones y roles que no les corresponden los cuales inciden negativamente en su desarrollo personal.

Sin embargo, es importante resaltar que en los resultados se ve como la exigencia del imaginario social de familia instituye una serie de elementos en los sujetos que las conforman, por lo cual esta idea de familia que ha sido culturalmente construida está fuertemente atravesada por el sistema económico y político que vive la familia actual, separándolo en ocasiones de lo planteado sobre afectividad, ciclo vital y estructura familiar, incidiendo notablemente en las motivaciones de las personas en el momento de conformarla.

\section{El "Autoritarismo" como Pauta de Crianza}

Otro de los imaginarios que coincide en las Investigaciones, el "autoritarismo" como pauta de crianza de los padres hacia los hijos en mayor expresión con jóvenes adolescentes. Los padres y 
madres consideran que aportan de forma significativa en su formación a través del castigo físico, imposición de límites a través de reglas de disciplina que muchas oportunidades se realizan a través de violencia física y verbal, esto genera que se visualicen frente a sus hijos como muy exigentes, poco sensibles y compresibles de sus deseos y necesidades afectando su desarrollo emocional (Moreno, Rodríguez \& Vega, 2012).

\section{Configuración en la relación de pareja}

Por último, en el contexto de los imaginarios, los resultados evidencian cómo se naturalizan los círculos de violencia traducidos en agresión y maltrato, con mayor tendencia en el género femenino, ya que los consideran como tensiones normales que amenazan la relación de pareja y la familia, pero que deben persistir para mantener la conformación de la familia. "Las parejas manifestaron, tener las necesidades básicas satisfechas a pesar de que se presentan agresiones y maltratos, pero se sobrepasan por el amor que existe" (Montenegro \& Ramírez, 2011). Esto evidencia lo instituido del imaginario de Familia complementado con el imaginario que el "amor lo puede todo", al respecto, en el 2015, según Medicina Legal, 40.943 mujeres fueron víctimas de violencia de pareja. Los hallazgos evidencian que una característica, en la conformación de pareja, es el amor como indicador de satisfacción en la dinámica, el cual prevalece por encima de nivel educativo, nivel de ingreso y religión.

Otro aspecto importante se evidencia en los estudios y cuando pasa lo contrario a lo expuesto anteriormente y es cuando la pareja se separa, donde en la mayoría de los casos ausenta la figura paterna, genera- crisis internas en los hijos. Al respecto la investigación El Divorcio o la separación de los padres y las consecuencias en las hijas (Forero \& Rincón, 2013 pp. 57-58) describe, como estos impactos cruzan las fronteras del interior de la familia a los ámbitos escolares, sociales y personales expresados en su desarrollo emocional en el cual transitan sentimientos de tristeza, rebeldía y soledad, logrando un "quiebre" en las relaciones afectivas y comunicativas al interior de la dinámica familiar. Al respecto se argumenta:

Es claro que cualquier quiebre matrimonial, perjudica enormemente la relación de la familia. Esta, en pocas palabras, se quiebra. Ya no seguirá siendo la misma. Pero la obligación de aquellos padres separados, no termina con su ruptura. Su amor y cuidado, asimismo como la formación de los hijos, no termina ahí. Ya que ellos son prioridad número uno, de todos los padres de familia, para con sus hijos. (Maturana \& Varela, 1990, pp. 67-70).

Desde el estudio realizado con niñas que viven con padres separados por (Arias et. al., 2015, p. 57) se evidencia en los resultados cómo la situación de divorcio entra en conflicto al ejercer el rol de autoridad como padres, al no querer lastimar a sus hijos lo cual incide en permisividad e inadecuadas pautas de crianza.

\section{Núcleo de Conocimiento: El Afecto en la relación de Padres y sus hijos en "Estado de Emergencia"}

Este núcleo de conocimiento, aporta a la Unidad de Significación, de Educación para la Socioafectividad- la cual es considerada en la Especialización (Fundación Universitaria Monserrate, 2009, p. 64), es un campo en lo académico con un objeto de estudio aún delimitado en incipiente, e importante para la familia donde se "van instituyendo, co-construyendo, el mundo de los afectos, sentimientos y pasiones ligados con el sistema cultural-simbólico en el que se privilegian unos y se rechazan otros, según los ideales de las formas sociales de cada momento histórico y su contex- 
to”. En este sentido, los hallazgos de este núcleo posibilitaran las miradas frente a las necesidades contemporáneas que permiten problematizar el objeto de estudio.

Siguiendo los resultados, de las investigaciones se constata que el Afecto en la relación de padres de hijos se encuentra en estado de "Emergencia", lo cual significa que se necesita de atención de forma urgente en el contexto de la orientación y la Educación Familiar y ancla un punto en la mirada del cualificador de Sociafetividad, el cual concibe desde la perspectiva de eje formador en la Especialización de Educación y Orientación a la Familia como "el sistema de relaciones fundamentalmente afectivas, presente en todas las culturas, en el que el ser humano permanece largo tiempo, y no un tiempo cualquiera de su vida, sino el formado por sus fases evolutivas cruciales" (Nardon, Gianaotti \& Rocchi, 2003, pp. 38-39).

Al respecto Ortiz, Fuentes y López (citados en Palacios, Marchesi \& Coll, 1990, pp. 165-166) afirman que: "[...] el vínculo emocional más importante al menos en la primera infancia, es el apego, el vínculo afectivo que el niño establece con una o varias personas del sistema familiar". Este vínculo de apego que permite y ofrece al niño una serie de recursos emocionales que les puede otorgar la habilidad de construir su autoestima, experimentar sentimientos de protección, de seguridad, de reconocimiento, de aceptación y de sentir que son amados, importantes para sus padres, por esto es necesario fortalecer vínculos afectivos, los sentimientos y los lazos de amor desde el momento de nacer y particularmente vivirlos en la primera infancia.

Se encuentra en los estudios alta coincidencia, cómo los padres y madres manifiestan ser poco afectivos con sus hijos, en este contexto se materializa el imaginario de que no es necesario al cumplir con las necesidades básicas "se suple lo afectivo con lo material" y originando roles de padres y madres distantes y proveedores. Este fenómeno gesta diferentes problemáticas como, la falta de comunicación, la interacción, la soledad y por su puesto el maltrato en varias expresiones.

Otro de los hallazgos en este mismo contexto, evidencian cómo las familias generan estilos de comunicación autoritarios- acusadores- estrictos y hostiles (Rol acusador: uno los patrones de comportamiento de cuando alguien se somete a los efectos negativos de la tensión y el estrés: aplacar, culpar, calcular y distraer (Satir \& Murray, 2002), en el que prevalece el rol de superioridad, logrando anular cualquier posibilidad de expresión de afecto, distanciamiento de los padres hacia los hijos, tensionando la interacción familiar y la decisiones de algunos padres y madres de delegar la función socializadora de sus hijos a los abuelos y abuelas o en la modalidad de "institucionalización".

Según los estudios realizados en niños, niñas jóvenes institucionalizados, ellos reconocen la importancia del vínculo afectivo y la necesidad de reencontrarse con sus familias de origen para realizar procesos en este caso de rehabilitación de consumo de SPA, al respecto argumentan Ríos y González (1984, pp. 98-99), quienes desde su teoría nos muestran que:

La familia se convierte en la más importante red de apoyo social para las diversas transiciones vitales que han de realizar durante su vida [...] La orientación familiar fortalece las capacidades, refuerza los vínculos que unen los miembros de un sistema familiar para que todos puedan alcanzar los objetivos que tiene la familia como agente o como institución educativa.

Otro elemento encontrado al interior de las investigaciones estudiadas, dentro la familia son las situaciones que pueden influir negativamente sobre nuestros jóvenes o su proyecto de vida; quienes por circunstancias adversas se ven enfrentados a ingresar en ocasiones coaccionados o influenciados, al consumo de sustancias 
psicoactivas. Datos encontrados en los resultados del estudio presentado en los jóvenes farmacodependientes, realizado por la fundación Shekina, Gachancipá, Cundinamarca (Barrios \& Otálora, 2013); en el cual los jóvenes manifestaron la importancia de los lazos afectivos a nivel familiar y sentir, la necesidad de reencontrarse con su hogar de origen, "su familia”. Este proceso les permitirá tomar conciencia de su condición y a su vez, generar compromisos con su núcleo familiar. Es así que esta expresión de afecto y la necesidad de restaurar el vínculo afectivo que recibe en su hogar, son los primeros pasos para asumir la situación y afrontar el proceso de rehabilitación en la fundación, acompañada -claro está- de la disposición y la motivación de su familia para resignificar su vida y así, asumir los nuevos retos que le impone la sociedad, tomando el propio control de su vida (Barrios \& Otálora, 2013, p. 97).

Para estos jóvenes, los lazos afectivos que reciben dentro de su núcleo familiar son de vital importancia dentro del proceso de rehabilitación ya que son parte de la motivación para buscar ayuda y restructurar el proyecto de vida.

De acuerdo a lo anterior y a los análisis de los estudios, se aducen algunas posibles causas:

Ausencia del Rol Materno: Cuando este fenómeno se inscribe al interior de las familias (Moreno, Rodríguez \& Vega, 2012), se evidencia la necesidad de la multiplicación de roles, habita la configuración de abandono acompañado de sentimientos, de rabia, dolor y miedo en los hijos quienes en con acciones buscan una acción de castigo a la figura femenina.

Condiciones Socio- Económicas: se identificó que los padres y las madres no dedican el tiempo necesario, para estar con sus hijos e hijas por la necesidad de trabajar en jornadas largas para la consecución de la satisfacción de necesidades básicas, lo cual es una realidad contemporánea.

Violencia Aprendida: Los padres y madres, argumentan que les cuesta expresar sus sentimientos a acusa de dolores no que han sido tramitados, sus vidas han estado inmersas en círculos de conflicto, que consolidaron la naturalización de tipos de violencia aprendidos.

\section{Núcleo de Conocimiento: Adolescencia "Un terreno lleno de Incertidumbre”}

En este núcleo de conocimiento encontró aspectos constitutivos como: la relación de padres e hijos adolescentes es una "lucha" de contrarios, en el cual los hijos e hijas adolescentes, se tensionan con los estilos educativos que utilizan sus padres, tal como lo refiere uno de los estudios: "[...] los jóvenes se sienten incomprendidos, al sentir que no son reconocidos ni respetados en los cambios físicos y psicológicos dados por la etapa del ciclo vital que atraviesan" (Alarcón et al., 2013 p. 1).

En este sentido los hallazgos muestran que los padres y madres tienen miedos ocultos, que se expresan a través de pautas de crianza autoritarias o permisivas que tensionan la comunicación e interacción y generan actitudes de rebeldía en los hijos. Sumado a esto el castigo también se considera como una forma de buena "educación". "A veces digo ¿será que no soy buena mamá? A veces Juan David me gana y es horrible, porque no me gusta pegarle, pero yo a veces siento que mi hijo me gana" (Madre entrevistada, citada en Alarcón et al., 2013 p. 18).

En esta línea de argumentación, los hallazgos evidencian la necesidad de la formación y orientación para padres.

Otro territorio de incertidumbre, es la sexualidad de los adolescentes, el cual habita en la familia en forma silenciosa y según los hallazgos las familias de este grupo poblacional evidencia que el tema es un "tabú" el cual no se sabe cómo abordar en cuanto no se tiene claridad educativa para hacerlo y existen marcos pre-concebidos desde pautas de crianza, imaginarios y patrones culturales 
en relación con la formación sexual, que generan a los padres inseguridad al tratar el tema; de igual forma consideran que al conversar con sus hijos de sexualidad, promueven el deseo sexual y curiosidad que da pauta a inicio de la actividad sexual.

Lo anterior suscita que la educación sexual de forma consciente o inconsciente por parte de los padres se delegue a las instituciones educativas, compañeros, medios de comunicación y redes sociales, donde se configura la sexualidad reducido a las relaciones sexuales.

En este sentido, los jóvenes afirman que es muy difícil hablar de con los padres de estos temas, por tal razón, se consideran que no tienen gran conocimiento en Educación Sexual. Se puede inferir entonces que existen barreras desde la comunicación de padres hacia jóvenes que abren brechas causantes de otras situaciones tales como inicio de actividad sexual a temprana edad, embarazos en edad adolescente, los cuales a su vez traen dificultades implícitas como el cambio del proyecto de vida, transferencia de pautas de conflicto y violencia hacia sus hijos.

Relacionado a lo anterior, en los estudios elaborados por Cadavid, Castrillón, Castro y Vivas (2011, p. 120) se encuentra un aspecto que emerge y son las relaciones afectivas en adolescentes, las cuales se describen como "entrega absoluta por el otro", con la concepción de hacer feliz al otro por encima de los conflictos, el otro se convierte en la única fuente de afecto, seguridad y confianza. Al analizar dichos estudios se evidencian frases que retoman el tema como: "si logro que alguien me ame, todo es posible, tengo asegurada mi felicidad eterna" y "cuando estoy enamorado, el mundo cambia mágicamente", "si no tengo a otro como mi pareja, mi mundo está vacío y carente de sentido". Esto coincide con lo siguiente: allí hay una alta incidencia sobre los imaginarios de los jóvenes que se enmarcan en un patrón sexista, donde predomina la visión machista, respecto al género y los roles de género, y se evidencia en sus imaginarios una mujer predominantemente reservada, respetuosa, cariñosa, femenina, débil y sensible (Fonseca, Rojas \& Román, 2012, p. 98).

Dando cumplimiento al segundo Objetivo Especifico se describen los:

Segundo Objetivo Específico: Aportes teóricos de las unidades de significación del objeto de conocimiento a los Estudios de investigación periodo (2010-2015)

La formación en cada una de las Unidades de Significación y las teorías utilizadas en cada una de las investigaciones permitieron el acercamiento y la comprensión de las dinámicas familiares y por supuesto, el abordaje de los procesos de investigación en cada uno de los cualificadores de formación y los modos de establecer procesos de orientación y relación de ayuda. Estas son las teorías abordadas:

\section{Educación y Orientación desde la comunicación}

En esta Unidad de significación, se encontró que las investigaciones abordaron las teorías por la especialización como: la Comunicación y los patrones de Comunicación, la dinámica familiar en el campo de la comunicación, la conceptualización pareja y la comunicación, la dinámica familiar en el campo de la comunicación.

\section{Educación y Orientación desde la organización}

Las teorías abordadas en los estudios fueron: la familia, desde lo estructural, lo funcional y lo evolutivo, los estilos educativos parentales, relación de padres e hijos, autoridad y poder. En este sentido, se comprenden las creencias, relaciones e interacciones de cada uno de los miembros y como configuran desde allí procesos de socialización. 


\section{Educación y orientación en lo afectivo}

Se encontraron las siguientes teorías, abordadas en los estudios fueron: construcción de vínculos afectivos, desarrollo psicológico en la primera infancia, sentimientos y emociones.

De igual forma, estos aportes han potenciado el pensamiento profesional de los estudiantes de la Especialización en el marco de la orientación; también ha fomentado el interés por la formación investigativa en el contexto de la familia.

Se evidencia entones que el aporte de la Especialización desde su formación a la comprensión de las realidades de las familias participantes en cada uno de los estudios realizados por los estudiantes entre el periodo 2010- y 2015, al encontrar en los hallazgos nuevas necesidades para la formación y orientación familiar en el contexto contemporáneo.

Dicho esto, se proponen entonces algunas nociones e ideas fuerza a manera de conclusión, que emergen de los hallazgos encontrados, las cuales posibilitan fortalecer las miradas en el proceso de formación en la Especialización de Educación y Orientación Familiar.

\section{Conclusiones}

\section{Nociones - La Familia un espacio de convergencia}

Los resultados evidencian cómo la familia, se configura a partir de unos momentos históricos, que han institucionalizado su forma de ser y de habitar en el mundo; en este contexto, plantea el diseño coordenadas de acuerdo sus imaginarios, que convergen en los sentidos, los significados, los lenguajes, las emociones, los pensamientos, los saberes, las relaciones, las interacciones y las acciones.

De acuerdo a lo anterior, ver a las familias como un espacio de convergencia, significa conversar y comprender los cambios constantes de éstas, significar sus potencialidades, las tensiones y las necesidades de orientación, lo cual puede hacer "terruño", es decir, generar pertenencia en el territorio en la especialización y de los futuros especialistas.

\section{Imaginarios que configuran tensiones contemporáneas}

Los imaginarios encontrados en la investigación, evidencian las tensiones contemporáneas; entonces, es vital reflexionar y anclar la mirada, en cada una de las unidades de significación en categorías específicas de la relación familia - escuela, lo cual significa abordar y orientar los agentes educativos para la comprensión de sus dinámicas y potenciar saberes de los padres, que incidan en los procesos educativos de sus hijos.

\section{Socio afectividad familiar}

El desarrollo emocional y su impacto en el desarrollo humano, es un aspecto de "fina mirada" en los procesos de orientación y educación familiar; pues como evidencia en los hallazgos, hay una "emergencia" en relación con educación para la expresión del afecto al interior de las familias como "conexión de cuidado" consigo mismo y con el otro.

\section{Adolescencia un terreno "Incomprendido"}

Los datos evidencian la necesidad prioritaria de investigar y comprender este ciclo vital, el cual es un terreno fértil para la incomprensión, la violencia, el consumo de SPA; los jóvenes no se sienten comprendidos y los padres no saben cómo comprenderlos. En este sentido, es fundamental indagar sobre las potencias, los saberes y las necesidades de los y las adolescentes desde perspectivas neurológicas, educativas, sociales y familiares. 


\section{Referencias}

Alarcón, G., Castro, M., Mayorga, A. H., Montoya, L. E. \& Layton, L. (2013). Los estilos educativos parentales como pilares de los procesos socializadores en las familias. (Tesis de Especialización). FUM. Bogotá.

Arias, B. J., Gámez, J. C., Gómez, Y. P. \& Guerrero, A. P. (2011). Incidencia de los procesos relacionales y la función socializadora de la familia en las habilidades comunicativas de los niños y niñas de 3 a 4 años. (Tesis de Especialización). FUM. Bogotá.

Asencio. G., \& Cardozo. E. L. (2015). Docentes dinamizadoras de la Especialización de Educación y Orientación Familiar de la Fundación Universitaria Monserrate. Bogotá: FUM.

Amaya, P. D., Bautista, M., Ortiz C., Bayona, M., Bejarano., Rodríguez. Y A. Benito, Murcia, N., Cardona, C., Castiblanco, A.S., Collazos, Mancilla. L., Cubillos. P.S., González, L. Y. A., González, Medina. D., Jiménez. L., Jiménez. T.G., Malangón. J., Martínez. M. J., Melo. R. L., Méndez. D., Miranda. G. A., Niño.L.V., Ortiz, G. S., Ramírez. A.G., Santamaría. C.L., Tunjano, S. L., Valderrama. U.D., Vargas. R. A., \& Virguez. V.P. (2015). Auxiliares de investigación, estudiantes Corte 81 de la Especialización de Educación de Orientación y Educación Familiar de la Fundación Universitaria Monserrate. Bogotá: FUM.

Barrios Cediel, M. Á., \& Otálora López, M. I. (2013). Sistematización de la experiencia con jóvenes farmacodependientes, en la Fundación Shekina, Gachancipá, Cundinamarca. (Tesis de Especialización de la Fundación Universitaria Monserrate. FUM:.Bogotá.

Bolívar, A. (2006). Familia y escuela: dos mundos llamados a trabajar en común. Revista de educación. (339). 119-146. España: Universidad de Granada. Cadavid, M., Castrillón, R., Castro, M., Vivas, R. (2011). Imaginarios acerca de paternidad/maternidad en adolescentes del grado noveno de la ins- titución educativa distrital integrada de Fontibón, Jornada mañana, Sede A, a través de la implementación del programa ¿Bebé?, ipiénsalo bien! (Tesis de grado). Especialización de la Fundación Universitaria Monserrate. FUM: Bogotá.

Castoriadis, C. (1989). La institución imaginaria de la sociedad. Barcelona: Tusquets.

Forero, M, \& Rincón, G. (2013). El divorcio o la separación de los padres y las consecuencias en las hijas. (Tesis de especialización de la Fundación Universitaria Monserrate. Bogotá: FUM.

Fonseca, R. D. J., Rojas, O. L., \& Román, D. J. (2012). Imaginarios que tienen sobre la sexualidad los adolescentes de grado $9^{\circ}$ de las instituciones monseñor Agustín Gutiérrez. Bogotá: FUM.

Fundación Universitaria Monserrate (2009). Documento Marco Renovación Registro Calificado Especialización en Educación y Orientación Familiar. Bogotá: FUM.

Galeano M. (2004). Diseño de proyectos en la investigación cualitativaa. Bogotá: Fondo Editorial Universidad Eafit.

González, M. A. (2003). Los paradigmas de investigación en las Ciencias Sociales.

Infante, J. \& Alayón, P. (2013). Imaginarios sociales de la construcción de familia de los padres y madres jóvenes del sector de Alfonso López de la localidad de Usme y cómo lo vinculan a su proyecto de vida. Bogotá: FUM.

Lozano, F. (2015). Territorio en la Escuela. Conversatorio en el marco de Estudio IDEP. Bogotá: Instituto para la Investigación Educativa y el Desarrollo Pedagógico.

Nardone, G, Giannotti E \& Rochhi Rita. (2012). Modelos de Familia. 5a. Ed. España: Editorial Herdel.

Maturana, H., \& Varela, F. (1990). El árbol del conocimiento. Madrid: Debate.

Montenegro Galindo, S., \& Ramírez Moreno, M. C. (2011). Evaluación del impacto social del programa "Atención e intervención psicosocial a parejas expuestas a violencia conyugal" en el componente 
psicosocial, usuarias de la comisaria de familia de Cajicá en el primer periodo del año 2011. (Tesis de grado). Especialización de la Fundación Universitaria Monserrate. FUM: Bogotá.

Moreno Ríos, M. G., Rodríguez Martínez, D. M., \& Vega, O. H. (2012). Imaginarios sobre pautas de crianza de los padres de familia o tutores grado tercero del Liceo Rodrigo Arenas Betancourt. (Tesis de grado). Especialización de la Fundación Universitaria Monserrate. FUM: Bogotá.

Moreno, A. Ospina, L. A.M. Velázquez, P. \& Velandia, B. (2012). Familia y Escuela: interés permanente en procesos formativos. (Tesis de grado). Espe- cialización en Educación y Orientación Familiar. FUM: Bogotá.

Sampieri, R, Fernández, C, Baptista, P. (2010). Metodología de la investigación. 5a Ed. México: McGraw Hill.

Satir, V, \& Murray, B. (2002). Dos enfoques de la Terapia Familiar. Bogotá: Universidad Nacional.

Palacios, J, Marchesi, Á, \& Coll, C. (2009). Desarrollo Psicológico y Educación. Tomo I. Madrid: Alianza Editorial, S.A.

Ríos, González, J. A. (1984). Orientación y Terapia familiar. Madrid: Fundación Instituto Ciencias del Hombre. 